\title{
Risikokompetenz in der Schule lernen
}
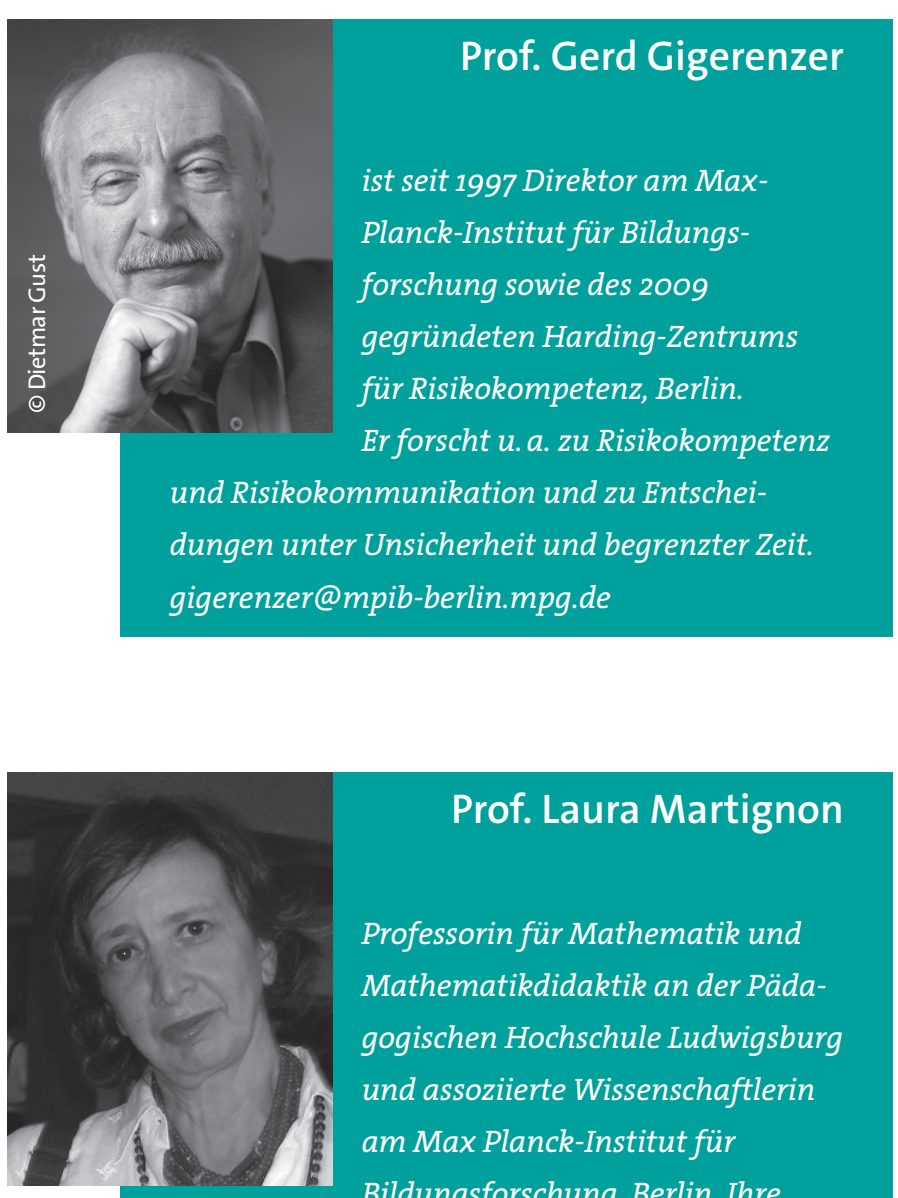

Prof. Laura Martignon

Professorin für Mathematik und

Mathematikdidaktik an der Päda-

gogischen Hochschule Ludwigsburg

und assoziierte Wissenschaftlerin

am Max Planck-Institut für

Bildungsforschung, Berlin. Ihre

Forschungsschwerpunkte liegen im Bereich

Didaktik der Stochastik in der Grundschule und

der Entscheidungsfindung unter Unsicherheit.

martignon@ph-ludwigsburg.de
Dieser Beitrag beruht zum Teil auf Gigerenzer, G. (2013). Risiko. Wie man die richtigen Entscheidungen trifft. Gütersloh: Bertelsmann. Wir danken Maren Wöll für das Editieren des Artikels.
Dieser Beitrag beschreibt Situationen, bei denen Informationen zu Risiken unentbehrlich sind. Diese Informationen können verständlich ausgedrückt werden oder auch unverständlich, mit schwierigen Formeln und undurchsichtigen Graphiken. Hier wird für sehr einfache, transparente Repräsentationen plädiert, die das Verständnis fördern. Nach einigen Beispielen aus der medizinischen Praxis wird eine Lanze für die Einführung erster Risikobegriffe in der Grundschule gebrochen.

\section{EINLEITUNG}

Im Oktober 2009 erschien in der Zeitschrift Nature ein vierseitiger Bericht mit dem Titel „Risk School“. Dort wurde die Frage diskutiert, ob Menschen jemals lernen werden, mit den Risiken einer modernen technologischen Welt kompetent umzugehen, oder ob sie damit hoffnungslos überfordert seien. Auf der einen Seite sahen es die Anhänger des „Nudging“-Programms (Thaler \& Sunstein, 2008) als erwiesen an, dass Menschen die nötige Portion Rationalität fehlt und sie immer wieder die gleichen Fehler beim Denken und Handeln machen. Da diese Fehler ebenso wenig korrigiert werden können wie etwa optische Illusionen, sollen Regierungen oder andere Autoritäten die Menschen sanft - d.h. ohne Zwangsmaßnahmen - in die richtige Richtung lenken. Die Methoden des „Nudging“ sind nicht neu, sie werden seit langem von Werbung und Marketing eingesetzt. Das Ergebnis ist eine Form von weichem Paternalismus, der nicht verbietet oder belohnt, sondern unser Verhalten untergründig steuert. Die Cameron-Regierung in Großbritannien und die Obama-Regierung in den USA haben solche NudgingProgramme eingesetzt.

Die andere Seite, für die die Autoren dieses Beitrags stehen, argumentiert, dass mangelnde Risikokompetenz im Unterschied etwa zu optischen Illusionen nicht genetisch verankert und damit quasi nicht zu korrigieren ist, sondern dass jeder Mensch Risikokompetenz erwerben kann. Die Methoden dafür sind bekannt (siehe unten), werden aber in der Schule bisher so gut 
wie nie gelehrt, und in der Folge können die meisten Menschen Risiken nicht verstehen. Also müssen wir die Schule - und die Lehrerausbildung - revolutionieren. Wir lehren unseren Kindern immer noch die Mathematik der Gewissheit also Algebra, Trigonometrie, kaum aber die Mathematik der Ungewissheit, wie statistisches Denken. Dies führt dazu, dass die meisten Ärzte die Ergebnisse von Krebsfrüherkennungs-Tests nicht verstehen und die meisten Richter und Rechtsexperten DNA-Tests nicht richtig beurteilen können (Gigerenzer 2002; 2013). Solange wir nicht den Mut haben, die Schule zu revolutionieren, bleiben paternalistische Ansätze wie das „Nudging“ die einzige Alternative. Als Philosophie für das 21. Jahrhundert kann das nicht überzeugen. Wir wollen nicht, dass künftige Generationen ihr Leben nur dann bewältigen können, wenn sie von anderen gelenkt und angestoßen werden. Unsere Kinder haben Besseres verdient. Vor Jahrhunderten konnte sich kaum jemand vorstellen, dass es Gesellschaften geben könnte, in denen fast jeder lesen und schreiben kann. Solche Gesellschaften gibt es inzwischen. Heute können sich nur wenige eine Gesellschaft vorstellen, in der fast jeder risikokompetent ist. Aber auch solche Gesellschaften wird es bald geben, wenn wir uns dafür einsetzen. Um dahin zu gelangen, müssen wir lange vor dem Medizin- oder Jurastudium beginnen. Selbst kleine Kinder können Risiken und Wahrscheinlichkeiten verstehen, wenn man sie ihnen spielerisch vermittelt. Früherziehung in Risikointelligenz wird wesentlich dazu beitragen, dass eine neue Generation lernt, mit den Ungewissheiten des Lebens umzugehen, und damit den Paternalismus zu einem Gespenst der Vergangenheit zu machen.

\section{RISIKO IN DER MEDIZINISCHEN PRAXIS}

\section{Relatives und absolutes Risiko}

Kann die Art und Weise, wie Informationen zu bestimmten Risiken formuliert werden, hilfreich wirken? Katastrophal wirken kann sie auf jeden Fall, dazu ein Beispiel aus dem Bereich der Schwangerschaftsverhütung. Die britische Presse berichtete über die Ergebnisse einer Studie, nach der Frauen, die die Antibabypille der dritten Generation einnehmen, ihr Risiko einer Thromboembolie um 100 Prozent erhöhen. Thromboembolie kann zu schweren Schlaganfällen führen und tödlich sein. Tausende Britinnen brachen daraufhin bestürzt die Einnahme ab. In der Folge gab es eine Welle ungewollter Schwangerschaften und Abtreibungen. Was genau hatte die Studie, auf die sich die Nachrichten bezogen, gezeigt? Von je 7000 Frauen, die die Pille nicht eingenommen hatten, kam es bei einer Frau zu einer Thromboembolie; bei je 7000 Frauen, die die Pille eingenommen hatten, stieg die Anzahl von eins auf zwei. Das absolute Risiko stieg durch die Einnahme also um 1 von 7000 an, das relative Risiko erfuhr einen Anstieg um 100 Prozent (Gigerenzer, 2013). Die Art und Weise, wie eine Erhöhung (oder Verringerung) des absoluten bzw. des relativen Risikos angegeben wird, kann jeweils sehr verschiedene Reaktionen im „Innenleben“ der Adressaten auslösen. Schätzungen zufolge führte die Verbreitung der Nachricht, dass sich das Thromboembolie Risiko (relative Risikoerhöhung) um 100 Prozent erhöht hatte, letztlich zu mehr als 10000 zusätzlichen Schwangerschaftsabbrüchen. Besonders stark nahmen Abtreibungen und Geburten bei Mädchen unter 16 Jahren zu. Die britischen Frauen hatten also durchaus eine korrekte Information erhalten, allerdings in einer Form, die sie in vielen Fällen eine Entscheidung entgegen ihren eigenen Interessen und ihrer Gesundheit treffen ließ. Damit wurde diese Information in wahrsten Sinn heillos. Wie können Risiken transparent gemacht werden? Dazu gibt es ein einfaches Prinzip, das jeder lernen kann: Frage stets: Wie groß ist die absolute Risikozunahme?

\section{Analoge Repräsentation von Risiken: Brustkrebs}

Absolute Risiken kann man nicht nur numerisch, sondern auch analog darstellen. Dabei werden Symbole wie etwa Kreise verwendet, die jeweils ein Individuum darstellen. Durch die Eins-zu-eins-Setzung von Individuum und Symbol laden diese „Populationsdiagramme“ zur Identifikation ein. Der Betrachter kann sich besser vorstellen, eines dieser Individuen im Diagramm zu sein.

Betrachten wir das Populationsdiagramm in Abbildung 1, das auch in einem Artikel im The New England Journal of Medicine erschienen ist. Darin erklären Elmore und Gigerenzer (2005), wie das Risiko von Brustkrebs transparent kommuniziert werden kann. Die Frage ist, ob bestimmte Befunde bei Brustbiopsien das Auftreten von Brustkrebs nach 15 Jahren prognostizieren können. Verglichen mit Frauen in der allgemeinen Bevölkerung haben Frauen mit gutartigen Befunden aus einer Brust-Biopsie ein relatives Risiko von 1,27, an Brustkrebs zu erkranken; Frauen mit atypischen Befunden haben ein relatives Risiko von 4,24. Das entspricht einem relativen Risikoanstieg von $27 \%$ und $324 \%$. 
Die meisten Patienten und viele Ärzte verstehen diese Zahlen jedoch nicht. Abbildung 1 stellt dieselben Ergebnisse transparent dar: jeder Kreis symbolisiert eine Frau. Die dunklen Kreise stehen für Frauen mit Brustkrebs und illustrieren so die Entwicklung von „kein Brustkrebs“ zu „Brustkrebs“. Die oberen beiden Diagramme zeigen die „Baseline“: Von je 100 Frauen in der Allgemeinbevölkerung erhalten nach 15 Jahren 5 die Diagnose Brustkrebs. Die beiden mittleren Diagramme zeigen Frauen mit gutartigen Befunden. Nach 15 Jahren erhalten 6 Personen die Diagnose Brustkrebs. Damit wird klar, was das relative Risiko von 1,27 (entspricht einem $27 \%$ Risikoanstieg) bedeutet: etwa eine Frau mehr von je hundert erkrankt an Brustkrebs. Bei Frauen mit atypischen Befunden sind es nach 15 Jahren etwa 19 Frauen mit Brustkrebs - ein Anstieg von $324 \%$.

Jede Frau und jeder Arzt kann jetzt verstehen, was die relativen Zahlen bedeuten. Analoge Darstellungen von absoluten Risikoanstiegen können also helfen,
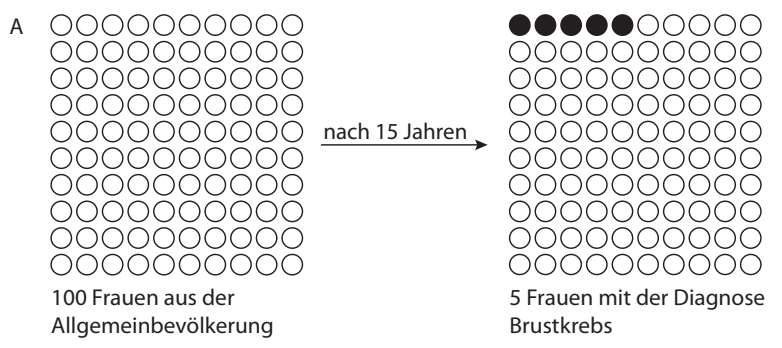

B
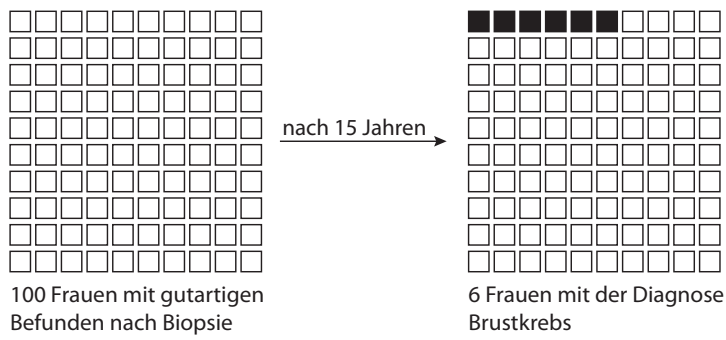

C

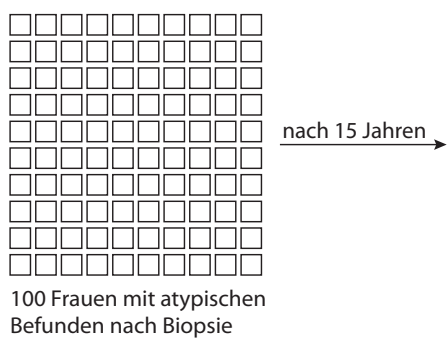

Situationen besser einzuschätzen und unnötige Ängste zu vermeiden.

\section{Natürliche Häufigkeiten und bedingte Wahrscheinlichkeiten: HIV-Tests}

Baumdiagramme gelten im Allgemeinen als gut verständliche und übersichtliche Darstellungen für eine große Familie von Problemstellungen in der Kombinatorik, in der Statistik und zur Klassifikation. Nicht jeder Baum ist allerdings intuitiv und unmittelbar verständlich. In Abbildung 2 sind zwei formgleiche Baumdiagramme nebeneinander gestellt. Der linke Baum enthält Angaben in relativen, der rechte in natürlichen Häufigkeiten (Gigerenzer \& Hoffrage, 1995). Warum ist dieser Unterschied wichtig? Eine frisch verheiratete und schwangere Frau in Kalifornien wurde von ihrem Arzt aufgefordert, einen Routine-HIV-Test durchführen zu lassen (Gigerenzer, 2013). Eine Woche später bestellte ihr Arzt sie wieder ein und teilte ihr mit, dass das Ergebnis des Tests (Western-Blot) positiv sei. Er beauftragte sie, ihrem Mann und ihrer Familie die Neuigkeit zu überbringen und schickte sie mit einem Stapel Internetausdrucke zum Leben mit HIV fort. Wie unschwer vorzustellen ist, brach für die Frau in diesem Moment eine Welt zusammen. Später stellte sich dann allerdings heraus, dass die Frau gar nicht HIV-infiziert war. Hätte sie von Anbeginn an diese Möglichkeit in Erwägung ziehen können?

Wie hoch ist die Wahrscheinlichkeit, dass eine positiv getestete Person auch wirklich HIV-infiziert ist? Das Baumdiagramm auf der linken Seite macht dazu beispielhaft die folgenden Annahmen: 0,01 Prozent der Menschen ohne Risikoverhalten sind HIV-infiziert. Von diesen werden 99,9 Prozent positiv getestet und entsprechend 0,1 Prozent negativ, Letztere fälschlicherweise. Bei den Nicht-Infizierten werden 99,99 Prozent korrekterweise negativ getestet und nur 0,01 Prozent fälschlicherweise positiv. Damit stehen die relevanten Angaben zur Verfügung und die Frage, wie hoch die Wahrscheinlichkeit ist, dass eine positiv getestete Person auch wirklich HIV-infiziert ist, kann beantwortet werden. Doch die meisten Menschen, Laien wie Experten, tun sich hier schwer. Eine typische Antwort von professionellen AIDS-Beratern lautet dann auch „99,9 Prozent“ (Gigerenzer, 2002), will heißen, die junge Frau hätte praktisch kaum Anlass gehabt eine Infektion anzuzweifeln - wie zunächst tatsächlich in diesem Fall geschehen.

Die rechte Seite in Abbildung 2 erzählt eine andere Geschichte. Die Geschichte ist schon deshalb anders, weil das Baumdiagramm ausschließlich natürliche Zahlen beinhaltet, die Häufigkeiten in einer angenom- 


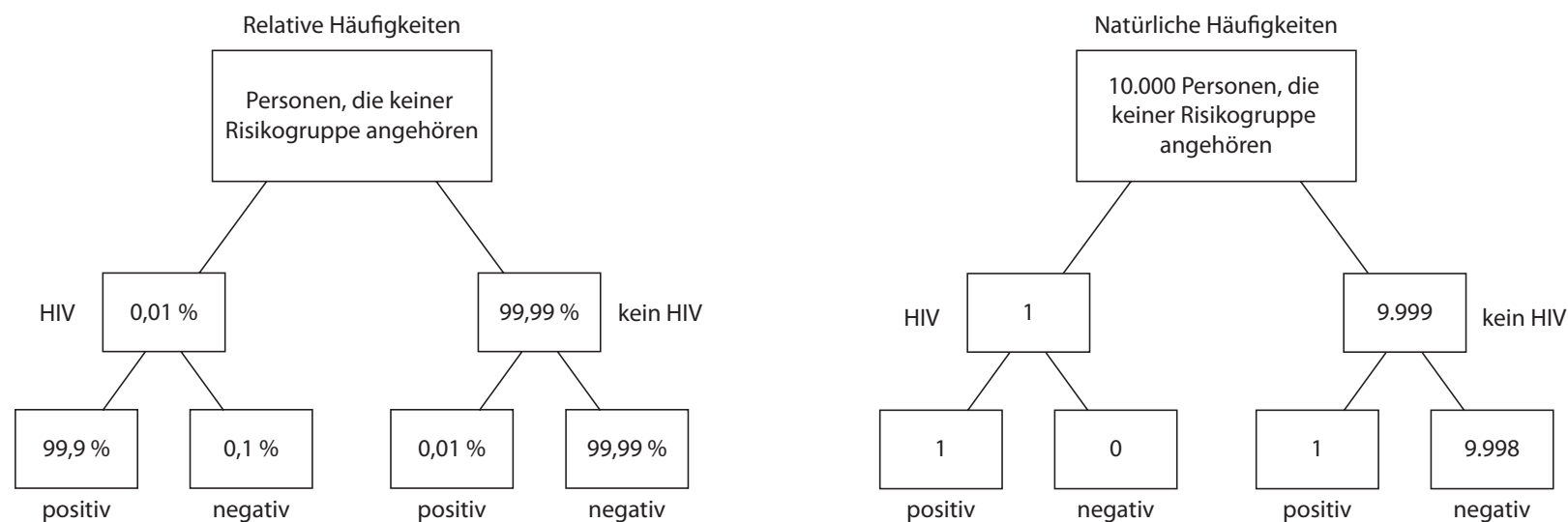

Abbildung 2. Zwei Arten von Häufigkeits-Bäumen mit relativen Häufigkeiten (links) und natürlichen Häufigkeiten (rechts). Die vier Werte unten links sind bedingte Wahrscheinlichkeiten (relative Häufigkeiten), die vier Werte unten rechts sind natürliche Häufigkeiten. Natürliche Häufigkeiten erleichtern das Verständnis des positiven prädiktiven Werts, hier der Wahrscheinlichkeit, dass eine Person HIV-infiziert ist, wenn sie ein positives Testergebnis erhalten hat. Diese ist hier $1 /(1+1)$, also $50 \%$.

menen Grundgesamtheit von 10000 Menschen darstellen, deshalb natürliche Häufigkeiten.

Die Situation für diese Grundgesamtheit von Menschen ohne Risikoverhalten ist die folgende: Einer unter diesen 10000 Menschen ist HIV-infiziert. Dieser Mensch testet in aller Regel positiv. Von den übrigen 9999 dann nicht HIV-infizierten Menschen testet ein weiterer Mensch ebenfalls positiv. Also ist nur einer der beiden positiv getesteten Menschen tatsächlich infiziert. Hätte die junge Frau aus Kalifornien sich in dieser 50-50 Situation gesehen, hätte sie sich nicht schon vor vollendete Tatsachen gestellt sehen müssen.

Nun haben wir uns erlaubt, die beiden Geschichten und Baumdiagramme Seite an Seite zu stellen. Sind die Angaben in den Diagrammen aber wirklich deckungsgleich? Nein, denn es handelt sich um eine Übersetzung numerischer Information. Diese Übersetzung möchte nicht konzept-getreu sein, sondern bietet eben gerade eine echte Alternative in der Darstellung. Natürliche Häufigkeiten sind keine relativen Häufigkeiten, da sie sich qua definitionem auf eine bestimmte Grundgesamtheit beziehen, deshalb leisten sie nicht das, was relative Häufigkeiten vermögen. Gleichzeitig leisten sie aber mehr, denn sie bieten einen festen Bezugs- und Ausgangspunkt für die Modellierung an, das kann hilfreich, vielleicht sogar heilsam sein, wenn Risiken eingeschätzt werden sollen. Kommt man bei korrekter Verrechnung der Information (Abb. 2) für die beiden Bäume zu unterschiedlichen Einschätzungen der Situation der jungen Frau? Nein. Kommen die allermeisten Menschen mit diesen beiden Darstellungen zu unterschiedlichen Einschätzungen der Situation der jungen Frau? Ja. Warum versteht man ein Testergebnis besser mit natürlichen als mit relativen Häufigkeiten? Die
Antwort lautet: Natürliche Häufigkeiten vereinfachen die Berechnung der Wahrscheinlichkeit P(HIV|pos) einer HIV-Infektion im Falle eines positiven Tests, wie die beiden Gleichungen in Abbildung 3 zeigen.

Der Vereinfachungseffekt der Darstellungen mit natürlichen Häufigkeiten wird auch offenbar, wenn sie mit einer gewöhnlichen Kontingenztafel verglichen werden. Die Häufigkeit des gleichzeitigen Auftretens von zwei binären Merkmalen oder Kategorien in einer Population wird dargestellt durch $2 \times 2$-Kontingenztafeln. Alternativ könnte das gleichzeitige Auftreten durch Bäume mit natürlichen Häufigkeiten wiedergegeben werden. Dabei wird jeder Baum mit natürlichen Häufigkeiten entweder durch die Reihen oder die Spalten der entsprechenden Tafel dargestellt. Somit können zwei verschiedene Bäume dieselbe $2 \times 2$-Kontingenztafel wiedergeben. Mittels natürlicher Häufigkeiten kann auch jeder Laie und AIDS-Berater schnell verstehen, wie sich $\mathrm{P}$ (HIV|pos) ändert, wenn ein Test eine andere Falsch-Positiv-Rate hat. Es existieren Schätzungen, denen zufolge diese Rate bei den besten Tests bei nur 1 in 250000 liegt. Wenn man dies auf die Situation in Abbildung 1 anwendet, dann ergibt sich ein Baum mit 250000 Personen, von

$$
\begin{gathered}
\frac{P(\mathrm{HIV} \mid \text { pos })=}{P(\mathrm{HIV}) P(\operatorname{pos} \mid \mathrm{HIV})+P(\text { keinHIV }) P(\operatorname{pos} \mid \text { keinHIV })} \\
P(\mathrm{HIV} \mid \text { pos })=\frac{0,01 \% \cdot 99,9 \%}{0,01 \% \cdot 99,9 \%+99,99 \% \cdot 0,01 \%} \approx 50 \% \\
P(\mathrm{HIV} \mid \text { pos })=\frac{1}{1+1}
\end{gathered}
$$

Abbildung 3. Die Formel von Bayes in zwei Formaten. 
denen 25 infizierte Personen erwartet werden, die mit an Sicherheit grenzender Wahrscheinlichkeit positiv testen, und eine nicht-infizierte Person, die ebenfalls positiv testet. Das heißt, $\mathrm{P}(\mathrm{HIV} \mid \mathrm{pos})$ ist hier $1 /(25+1)$.

\section{Ein schneller und effizienter Entscheidungs- baum für die medizinischen Diagnose}

Bäume mit natürlichen Häufigkeiten können ohne weiteres auf Situationen erweitert werden, in denen das gleichzeitige Auftreten von mehr als zwei binären Variablen betrachtet wird, etwa mehrere Tests für eine Bedingung (Martignon, Vitouch, Masanori \& Forster, 2003). Wird die Anzahl der Variablen aber hoch, dann werden Bäume komplex, weniger verständlich und auch weniger gut in der Vorhersage, da man zu viele Fehler macht bei der Schätzung der vielen bedingten Wahrscheinlichkeiten oder natürlichen Häufigkeiten. Die Lösung für diese Probleme ist Vereinfachung: effiziente Entscheidungsbäume (,fast-and-frugal trees“; Martignon et al., 2003). Das Beispiel in Abbildung 4 bezieht sich auf ein konkretes Problem: Eine Person mit schweren Schmerzen in der Brust wird in ein Krankenhaus eingeliefert. Die Ärzte in der Notaufnahme müssen entscheiden, ob der Patient in eine Herz-Intensivstation oder eine normale Station kommt.

Dies kann eine Entscheidung über Leben und Tod bedeuten, weil der Patient im Fall eines Herzinfarkts in der Intensivstation behandelt werden sollte, anderenfalls in einer normalen Station. Bei einer konkreten Studie in einem Krankenhaus in Michigan (Green \& Mehr, 1997) trat der Umstand hinzu, dass die Ärzte 90 Prozent aller Patienten mit schweren Brustschmerzen auf die Intensivstation schickten, die daraufhin überfüllt war. In der Folge nahm die Qualität der Behandlung ab, die Kosten stiegen. Außerdem erhöhte sich das Gesundheitsrisiko für die Patienten, da die Intensivstation einer der gefährlichsten Orte im Krankenhaus ist (Patienten können durch Viren sterben, die dort vorhanden sind). Wie entscheidet man nun, auf welche Station ein Patient kommt? Zunächst gilt es, eine Reihe bewährter Kriterien aufzustellen, aber dann muss ein Entscheidungsprozess entworfen werden, der ausreichend schnell und effizient ist. Effiziente Entscheidungsbäume (Martignon et al., 2003) haben einen Endpunkt auf jeder Ebene, was zu $n+1$ Ausgängen für binäre Bäume führt, wobei $n$ die Anzahl der Fragen oder Indikatoren ist.

Ein solcher, schneller und effizienter Baum besteht aus drei Fragen, eine davon ist eine zusammengesetzte Information, die sich auf bewährte disjunkte



Abbildung 4. Ein schneller und effizienter Entscheidungsbaum für die Verlegung auf kardiologische Intensivstationen (nach Green \& Mehr, 1997). Die ST-Segment-Veränderung ist eine Anomalie im Elektrokardiogramm; der Faktor NTG zeigt einen vormaligen Gebrauch von Nitroglyzerin gegen Brustschmerzen an, der Faktor MI myokardialen Infarkt, ST $\leftrightarrow$ und ST $₫$ kennzeichnen eine ST Segment-Blockade und T steht für T-Wellen mit Überhöhung oder Umkehrung.

diagnostische Indikatoren stützt (Martignon et al., 2003; Martignon, Katsikopoulos \& Woike, 2008; Gigerenzer, 2007). Er war sehr erfolgreich: Das ursprüngliche Datenmaterial des Krankenhauses in Michigan bestand aus 89 Aufnahmen (Martignon et al. 2003); keiner der Patienten, der auf eine normale Station kam, bekam einen Herzinfarkt. Anders gesagt, die Entscheidungsprozedur (Green \& Mehr, 1997) mündete nicht in falsch negative Entscheidungen, kein Infarkt wurde übersehen. Gleichzeitig kamen durch diese Prozedur nur 35 Patienten, die keinen Infarkt bekamen, in die Intensivstation, was einer Falsch-positiv-Rate von 35 von 74 entspricht - deutlich besser als die der Ärzte, welche ursprünglich bei etwa $90 \%$ lag. Alles in allem führte der Entscheidungsbaum zu einer sehr viel kleineren Zahl von Aufnahmen auf die Intensivstation und damit zu besserer Qualität bei geringeren Kosten.

\section{Haptische Repräsentationen anhand von Steckwürfeln}

Steckwürfel in allen Farben können erfolgreich eingesetzt werden, um statistische Sachverhalte konkret darzustellen. Mit Hilfe von Steckwürfeln (siehe Kapitel „Haptische Repräsentationen statistischer Information anhand von Steckwürfeln“) kann man sehr leicht eine Frage beantworten, die 70-80 Prozent der Gynäkologen in Deutschland nicht richtig beantworten 
können (Gigerenzer, 2013): Eine Frau nimmt an einem Brustkrebs-Screening teil und bekommt ein positives Mammografie-Ergebnis. Wie hoch ist die Wahrscheinlichkeit, dass sie Brustkrebs hat? Damit diese Frage beantwortet werden kann, müssen wir nur die Verknüpfungen betrachten, die einen weißen Steckwürfel (positives Testergebnis) haben. Sucht man die Verknüpfungen mit einem weißen Würfel aus der ScreeningUrne heraus, bekommt man 77 Verknüpfungen, 70 rot-weiße und sieben grün-weiße. Nur die, die einen grünen Würfel aufweisen, haben Brustkrebs. Anders gesagt, nur sieben von 77 Frauen mit einer positiven Mammografie haben tatsächlich Krebs.

\section{RISIKOKOMPETENZ ALS ZENTRALES THEMA DER SCHULE}

Der Umgang mit Risiken sollte aber nicht als abstrakte Stochastik gelehrt werden, sondern als eine Disziplin, mit der ganz allägliche Probleme gelöst werden können. Der Erfolg hängt dabei ganz wesentlich von einigen Grundprinzipien ab. Dazu gehört vor allem das problemorientierte Lernen:

Ausgangspunkt ist dabei ein Problem aus der Lebenswelt der Schüler, die dann nach einer Lösung suchen sollen. Dabei können und sollen Schüler Fehler machen und daraus lernen. Die Tatsache, dass es um Probleme aus der wirklichen Welt geht, motiviert die Schüler, die verwendeten Methoden werden dadurch in ihrem Gedächtnis verankert. Das Gegenteil zu dieser Vorgehensweise ist die weitverbreitete Praxis, mit der Lösung zu beginnen und den Schülern dann Probleme zu geben, auf die sich diese Lösung immer anwenden lässt. Weitere Prinzipien zur Verbesserung der Risikokompetenz sind bestimmte Vermittlungsmethoden für statistische Informationen. Wir haben hier einige graphische Darstellungen besprochen, einschließlich solcher, die wir als analog bezeichnen. Dabei zeigt sich auch, dass das Sprichwort „ein Bild sagt mehr als 1000 Worte" nicht immer wahr ist. Wie numerische Darstellungen, so sind auch manche graphische Darstellungen verständlich, andere hingegen führen den Betrachter durch ihre undurchsichtige Darstellung leicht in die Irre. Nicht explizit behandeln können wir hier die Interessenkonflikte, die häufig die Ursache dafür sind, dass Darstellungen toleriert oder bevorzugt werden, die für die meisten Verbraucher, Patienten und Bürger nachweislich nicht besonders anschaulich oder sogar undurchschaubar sind (Gigerenzer, 2002).
Wir leben heute in einer Welt, in der der technologische Fortschritt immer schneller voranschreitet, während das Verständnis für die Technologien und deren Risiken weit zurückbleibt. Viele Richter verstehen DNA-Evidenz nicht (Gigerenzer, 2002), viele Ärzte verstehen Gesundheitsstatistiken nicht und viele AIDS-Berater verstehen die Ergebnisse von HIV-Tests nicht (Gigerenzer, 2007; 2013). Menschen ängstigen sich vor Risiken, von denen höchstwahrscheinlich keine tödliche Gefahr ausgeht, wie genetisch modifiziertem Mais oder Pestizidrückständen auf Obst und Gemüse, statt vor Zigaretten und Autos, und Journalisten, die darüber berichteten, sind in der Regel nicht im Verstehen von Wahrscheinlichkeiten geschult. Entsprechende Fälle werden jeden Monat in der „Unstatistik des Monats“ dokumentiert: unstatistik.de (Bauer, Gigerenzer \& Krämer, 2014).

Wie wir zu Beginn dieses Artikels ausführten, sind manche Experten der Auffassung, dass Otto Normalverbraucher einen angeborenen Mangel an statistischer Denkfähigkeit hat und man wenig tun kann, um diesen Zustand zu ändern. Experimente mit Kindern beweisen das Gegenteil. Multmeier zeigte beispielsweise, dass schon die meisten Viertklässler Bayesianische Aufgaben lösen können (wie in Abb. 2), wenn man ihnen natürliche Häufigkeiten gibt, und Till (2014) zeigte, wie effektiv vier Stunden Intervention im statistischen Denken schon in der Grundschule sein können. Darüber hinaus helfen natürliche Häufigkeiten und verständliche Darstellungsformen gerade auch Kindern mit einer „Rechenschwäche“, das heißt mit einer ungewöhnlichen Lernschwäche beim Erwerb rechnerischer Fertigkeiten (Multmeier, Gigerenzer \& Wegwarth, 2011). Überraschenderweise konnten diese Kinder Aufgaben genauso leicht lösen wie andere Kinder. Alles weist darauf hin, dass der Schlüssel zu einer risikokompetenten Gesellschaft in der Schule liegt.

Ein Curriculum der Risikokompetenz, wie wir es uns vorstellen, sollte zentrale Themen und Fertigkeiten vermitteln. Zu den Themen gehören:

- Gesundheitskompetenz

- Finanzkompetenz

- Digitale Risikokompetenz

- Umweltkompetenz (Nachhaltigkeitsdiskussion)

Drei Fertigkeiten sind für jedes dieser Themen zentral: Statistisches Denken, Faustregeln (Heuristiken), Psychologie des Risikos.

Gesundheitskompetenz umfasst das Verständnis von statistischer Information von Ernährungsmitteln und 
von gewöhnlichen Krankheiten. Finanzkompetenz umfasst das Verständnis von Zins und Zinseszins, aber auch von Faustregeln wie „kaufe kein Finanzprodukt das du nicht verstehst.“

Digitale Risikokompetenz betrifft die Fähigkeit, digitale Medien zu kontrollieren statt von ihnen kontrolliert zu werden. Dazu ist es wichtig, dass junge Menschen verstehen, wie ihr Verhalten psychologisch von anderen beeinflusst wird. Und Umweltkompetenz betrifft das Verständnis von Langzeitrisiken, die durch menschliches Verhalten entstehen, aber auch ein Verständnis und Gefühl für die Abhängigkeit des Menschen von der Natur. Einzelheiten sind anhand von Beispielen in diesem Beitrag illustriert und in ausführlicher Form in Gigerenzer (2007) und Gigerenzer (2002; 2013) erläutert.

Um den kommenden Generationen Risikokompetenz zu vermitteln, müssen wir die Schule revolutionieren, und das möglichst sofort. Wenn diese risikokompetenten Kinder erwachsen sind, werden wir endlich Ärzte, Finanzexperten und Rechtsanwälte haben, die Ungewissheiten verstehen.

Und wir werden Kunden und Patienten haben, die sich nicht ins Bockshorn jagen lassen und wissen, welche Fragen sie zu stellen haben. Sie werden die Glieder einer Gesellschaft sein, die mit Risiken informiert und entspannt umgehen kann.

\section{WIE KONSTRUIERT MAN EINEN EFFIZIENTEN ENTSCHEIDUNGSBAUM?}

Martignon et al. (2003) und Martignon et al. (2008) haben ein systematisierendes Verfahren entwickelt, um effiziente Entscheidungsbäume zu konstruieren, die Diagnose bei der Aufnahme in die Herz-Intensivstation erleichtern. Ihr Ansatz basiert auf Werten wie Sensitivität, Spezifizität und der Quote korrekter Diagnosen. Durch systematische Analyse konstruierten sie verschiedene effiziente Entscheidungsbäume. Im Folgenden erklären wir ein Konstruktionsprinzip, das Herzinfarkte sehr genau vorhersagt (Martignon et al., 2008) und lediglich auf der Betrachtung der Quoten für korrekte Positiv- und Negativdiagnosen beruht (Martignon et al., 2003).

\section{Ordnung der Prädiktoren}

- Mit dem Prädiktor anfangen, der die maximale Quote korrekter Positivdiagnosen besitzt.

- Alle verbleibenden Informationen werden nach der Höhe der Quote korrekter Positiv- und Negativdiagnosen geordnet.

\section{Entscheidungsregel}

- In jeder Ebene wird der Zweig mit der größten Anzahl an Individuen zu einem Endpunkt eines Pfades; eine positive Bewertung der Information an dem endenden Pfad schickt die Patienten zur Herz-Intensivstation, eine negative in eine normale Station.

- Die nächste Information in der Informationsreihung wird dem Zweig mit der nächst kleineren Anzahl von Individuen zugeordnet.

- Fortfahren, bis alle Informationen in dieser Weise verarbeitet sind.

Effiziente Entscheidungsbäume können helfen, die Kommunikation von Risiken transparent zu gestalten. Sie haben den Vorteil, dass sie gewöhnlich eine begrenzte Anzahl von Prädiktoren und Pfaden nutzen (verglichen etwa mit vollständigen Bäumen), wodurch sie leichter zu verstehen, einzuprägen und mitzuteilen sind. Indes ist es auch wichtig, die Konstruktionsweise dieser Bäume anschaulich zu machen.

\section{HAPTISCHE REPRÄSENTA- TIONEN STATISTISCHER INFORMATION ANHAND VON STECKWÜRFELN}

Steckwürfelchen aus Plastik in allen Farben sind nützliche flexible Materialien im Mathematikunterricht der Grundschule.

Steckwürfeldarstellungen laden zum Nachmachen ein. Sogar Kinder im Alter von 9 Jahren und jünger können sich das mehrmalige Ziehen von Würfelpaaren aus einer Urne vorstellen (Kurz-Milcke \& Martignon, 2006; Martignon \& Kurz-Milcke, 2006; Martignon \& Krauss, 2009). Da die Verknüpfungen problemlos geordnet, zusammengefügt und aus der Menge von Steckwürfeln herausgenommen werden können, können die Populationen ganz einfach verändert werden. Diese Veränderungen 
müssen dabei nicht tatsächlich ausgeführt oder wahrgenommen werden, sondern können auch bloß imaginiert werden. Beispielsweise das Klassifizieren mittels einer bestimmten Farbe (zum Beispiel weiße Würfel in der besagten ScreeningUrne) bereitet auf das Verstehen einer bedingten Häufigkeit vor. Ist ein weißer Würfel gegeben, was ist dann die Häufigkeit von Rot oder auch Grün? Diese relativen Häufigkeiten können gezählt werden und aufgrund der anschaulichen Darstellung korrekt bewertet werden.
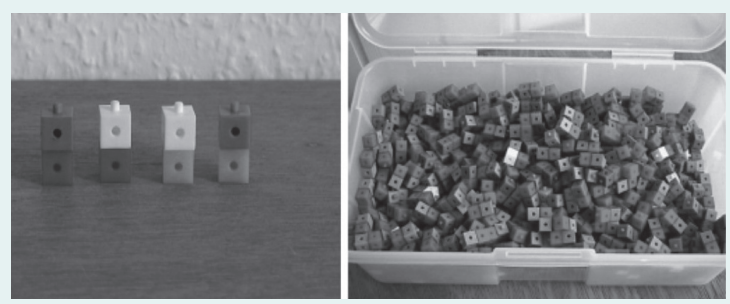

Abbildung 5. Verknüpfungen, die aus zwei Steckwürfeln bestehen. Die Farbe der Würfel wird zum Verschlüsseln von zwei binären Variablen verwendet, beispielsweise Gesundheitszustand (Krebs/kein Krebs) und Testergebnis beim Mammografie-Screening (positiv/negativ). Jede Verknüpfung verkörpert eine Person, in diesem Fall eine Frau, die an einem Mammografie-Screening teilnimmt. Rot heißt kein Krebs, Grün heißt Krebs, Blau bedeutet ein negatives Testergebnis und Weiß ein positives Testergebnis. Die Zuordnung der Farben erfolgte willkürlich.

Offenkundig können die Steckwürfel nach einer der vier Farben der Steckwürfel oder nach den vier Typen von Verknüpfungen sortiert werden (siehe Abb. 5, die die Sortierung gemäß bestimmter Verknüpfungen betreffen). Als letztes Beispiel nehmen wir an, dass die grünen Steckwürfel isoliert sind. Das führt zu einer relativ kleinen Anzahl von Verknüpfungen, da nur acht von 1000 Personen krebserkrankt sind.

Sieben davon sind mit einem weißen Steckwürfel gepaart und einer mit einem blauen. Daraus ergibt sich die Testsensitivität als relative Häufigkeit, da Frauen mit Brustkrebs (grün) ein positives Ergebnis beim Mammografie-Screening haben (weiß), nämlich in sieben von acht Fällen. Steckwürfeldarstellungen können beim Differenzieren zwischen bedingten Wahrscheinlichkeiten in Bezug auf verschiedene Referenzgruppen helfen. In unserem Beispiel haben wir uns auf krebskranke Personen konzentriert; wir könnten uns aber zu einem anderen Zeitpunkt z. B. mit den Personen beschäftigen, die positiv getestet wurden. Die Schwierigkeit mit symbolischen Darstellungen, nämlich das Durcheinanderbringen der bedingten
Wahrscheinlichkeiten von $\mathrm{p}$ (positiv|Krebs) mit denen von $\mathrm{p}$ (Krebs|positiv), entfällt bei der Arbeit mit Steckwürfeln.

\section{Literatur}

Bauer, T. K., Gigerenzer, G. \& Krämer, W. (2014). Warum dick nicht doof macht und Genmais nicht tötet: Über Risiken und Nebenwirkungen der Unstatistik. Frankfurt am Main: Campus Verlag.

Elmore, J. G. \& Gigerenzer, G. (2005): Benign breast disease The risks of communicating risk. New England Journal of Medicine, 353, 297-299.

Gigerenzer, G. (2002). Calculated risks: How to Know when Numbers Deceive You. New York: Simon \& Schuster. (UK version: Reckoning with Risk. Penguin).

Gigerenzer, G. (2007). Gut Feelings: The Intelligence of the Unconscious. New York: Viking. (UK: Allen Lane/Penguin).

Gigerenzer, G. (2013). Risiko: Wie man die richtigen Entscheidungen trifft. Gütersloh: Bertelsmann.

Gigerenzer, G. \& Hoffrage, U. (1995). How to improve Bayesian reasoning without instruction: Frequency formats. Psychological Review, 102, 684-704.

Green, L. \& Mehr, D. R. (1997). What alters physicians' decisions to admit to the coronary care unit? Journal of Family Practice, 45, 219-226.

Kurz-Milcke, E. \& Martignon, L. (2006). Lebendige Urnen und ereignisreiche Bäume: Überlegungen und Versuche zu einer Didaktik der Stochastik in der Grundschule. In J. Meyer (Hrsg.), Anregungen zum Stochastikunterricht, Bd. 3. (pp. 181-203). Hildesheim: Franzbecker.

Martignon, L. \& Kurz-Milcke, E. (2006). Educating children in stochastic modeling: Games with stochastic urns and colored tinkercubes. Proceedings of the ICOTS 7 Conference. C-443.

Martignon, L., Katsikopoulos, K. V. \& Woike, J. K. (2008). Categorization with limited resources. A family with simple heuristics. Journal of Mathematical Psychology, 52, 352-361.

Martignon, L. \& Krauss, S. (2009). Hands-on activities with fourth-graders: A tool box of heuristics for decision making and reckoning with risk. International Electronic Journal for Mathematics Education, 4, 117-148.

Martignon, L., Vitouch, O., Takezawa, M., Masanori, T. \& Forster, M. R. (2003). Naive and yet Enlightened: From Natural Frequencies to Fast and Frugal Decision Trees. In D. Hardman, L. Macchi (Eds.), Thinking: Psychological Perspectives on Reasoning, Judgement and Decision Making. Hoboken, NJ: John Wiley \& Sons, Ltd.

Multmeier, J., Gigerenzer, G. \& Wegwarth, O. (2011). How iconic representations foster children's Bayesian reasoning: studies in elementary school. Talk presented at the Annual Conference of the International Mind, Brain and Education Society, San Diego, CA, USA.

Thaler, R. H. \& Sunstein, C. R. (2008). Nudge. London, UK: Penguin.

Till, C. (2014). Fostering Risk Literacy in Elementary School. Mathematics Education, 9, 83-96. 